\title{
Sindrome de Wolfram: da Definição às Bases Moleculares
}

\begin{abstract}
RESUMO
A síndrome de Wolfram (SW) é uma condição neurodegenerativa progressiva de herança autossômica recessiva caracterizada pela presença de diabetes mellitus e atrofia óptica. Freqüentemente também estão presentes o diabetes insipidus e disacusia neurossensorial, explicando o acrônimo DIDMOAD (diabetes insipidus, diabetes mellitus, optic atrophy, deafness) pelo qual a síndrome é também conhecida. Além desses, outros comemorativos tais como bexiga neurogênica, ataxia, nistagmo e predisposição a doenças psiquiátricas podem também estar presentes. Em 1994 identificou-se no cromossomo 4p16.1 um dos genes responsáveis pela SW, que foi denominado WFS1 ou wolframina. Esse gene codifica uma proteína de 890 aminoácidos de localização no retículo endoplasmático. A função da proteína wolframina ainda não está completamente definida, porém sua localização no retículo endoplasmático sugere um papel na regulação da homeostase do cálcio, transporte de membrana ou processamento protéico. O entendimento de como alterações na função da wolframina resultam em diabetes e neurodegeneração é essencial para o desenvolvimento de terapias para prevenir ou atenuar as conseqüências da SW. (Arq Bras Endocrinol Metab 2006;50/5:839-844).
\end{abstract}

Descritores: Síndrome de Wolfram; DIDMOAD; WFS1; Wolframina; Diabetes mellitus

\section{ABSTRACT}

Wolfram Syndrome: From Definition To Molecular Bases.

Wolfram syndrome (WS) is an autosomal recessive progressive neurodegenerative disorder characterized by diabetes mellitus and optic atrophy. Diabetes insipidus and sensorineural deafness are also noted frequently, explaining the acronym DIDMOAD (diabetes insipidus, diabetes mellitus, optic atrophy and deafness) by which the syndrome is also referred. Additional manifestations such as atonic bladder, ataxia, nystagmus and predisposition for psychiatric illness may be present. The Wolfram syndrome gene, WFS1, was mapped to chromosome 4p16.1 by positional cloning. It encodes an 890-amino-acid polypeptide named wolframin. Although the wolframin function is still not completely known, its localization to the endoplasmic reticulum suggests it can play a role in calcium homeostasis, membrane trafficking and protein processing. Knowing the cellular function of wolframin is necessary for understanding the pathophysiology of Wolfram syndrome. This knowledge may lead to development of therapies to prevent or reduce the outcomes of WS.

(Arq Bras Endocrinol Metab 2006;50/5:839-844)

Keywords: Wolfram syndrome; DIDMOAD; WFS1; Wolframin; Diabetes mellitus

\author{
Maria Regina F. Ribeivo \\ Felipe Crispim \\ Márcio F.Vendramini \\ Regina S. Moisés
}

Disciplina de Endocrinologia, Universidade Federal de São Paulo/ Escola Paulista de Medicina (UNIFESP/EPM), São Paulo, SP.

Recebido em 09/12/05

Revisado em 04/04/06 Aceito em 18/04/06 
A SÍNDROME DE WOLFRAM (SW) é definida como uma condição neurodegenerativa envolvendo o sistema nervoso central, nervos periféricos e tecidos neuroendócrinos (1). É uma doença rara, progressiva e de herança autossômica recessiva, que foi primeiramente descrita em 1938 por Wolfram e Wagner (2). As características iniciais e essenciais para o diagnóstico são a presença de diabetes mellitus de início precoce e atrofia óptica. Esses critérios dão um valor preditivo positivo de $83 \%$ e valor preditivo negativo de $1 \%$ para a SW (3). Em geral, o diabetes mellitus desenvolve-se na primeira década de vida e a atrofia óptica na segunda década (3). Outros achados freqüentes, porém não obrigatórios, são o diabetes insipidus e surdez explicando o acrônimo DIDMOAD (de diabetes insipidus, diabetes mellitus, optic atrophy e deafness) pelo qual a síndrome é também conhecida. Diabetes insipidus ou surdez estão presentes em cerca de $51 \%$ dos casos, enquanto os quatro componentes cardinais estão presentes em apenas 13\% dos casos (3). Ainda, bexiga neurogênica, ataxia, nistagmo e predisposição a doenças psiquiátricas podem também ser componentes da síndrome, embora com menor freqüência. Apresentamos na figura 1 a história natural da doença.

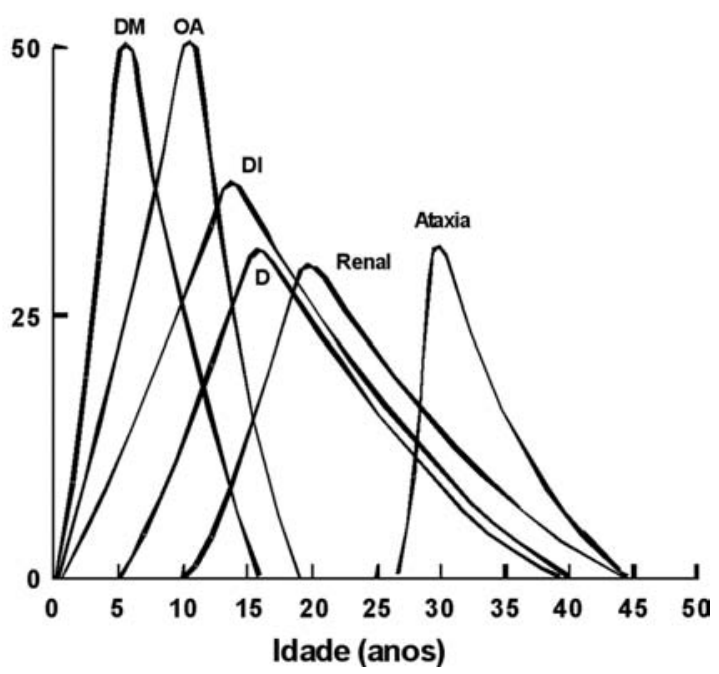

Figura 1. História natural da síndrome de Wolfram. O pico de cada curva representa a idade média de aparecimento das complicações, e a intersecção com o eixo da abscissa o intervalo de aparecimento.

DM: diabetes mellitus, AO: atrofia óptica, DI: diabetes insipidus, D: surdez, Renal: anormalidades do trato renal, Ataxia: anormalidade neurológicas. (Reproduzido de Barret $G$ e cols. (3), com permissão de Elsevier)

\section{DIABETES MELLITUS}

O diabetes mellitus é a primeira manifestação da SW na maioria dos casos reportados (4). A idade de aparecimento é precoce, em geral na primeira década de vida, mas pode ter início na idade adulta (5). É o resultado de uma deficiência de insulina de etiologia não auto imune (3). Estudos postmortem mostram uma perda seletiva das células $\beta$ pancreáticas com preservação das células produtoras de glucagon e somatostatina $(4,6)$. Entretanto, cetoacidose não é freqüente na apresentação inicial do diabetes ou durante a evolução $(4,7)$. Em relação aos antígenos do sistema HLA, alguns autores sugerem uma associação positiva com HLA-DR2, que é negativamente associado ao diabetes mellitus tipo 1 clássico $(8,9)$. Por outro lado, os pacientes com SW apresentam uma baixa prevalência dos antígenos DR3 e DR4, haplotipos freqüentes no DM tipo 1. Em geral, os pacientes são tratados com insulina, não havendo relatos de tratamento bem sucedido a longo prazo com agentes orais. Em relação às complicações crônicas do diabetes mellitus, uma baixa prevalência é reportada, especialmente de retinopatia e nefropatia, mesmo com controle metabólico inadequado $(3,4)$. Entretanto, em um caso por nós reportado previamente, verificamos a presença de retinopatia incipiente após 7 anos de diagnóstico da doença e controle metabólico inadequado (10).

\section{ATROFIA ÓPTICA}

A atrofia do nervo óptico é bilateral e progressiva, porém com velocidade variável de progressão. $\mathrm{O}$ início da diminuição da acuidade visual ocorre em geral na segunda década de vida, com idade média de 14 anos (11). O exame oftalmológico mostra palidez do nervo óptico com aumento do reflexo das artérias retinianas e angiofluoresceinografia e eletrorretinografia de campo geralmente normais. Os registros de potenciais visuais evocados revelam comprometimento na via máculo-occiptal (12). Estudo anátomo-patológico demonstra destruição axonal e desmielinização em todo o sistema óptico $(11,13)$. Outras alterações oculares menos freqüentes são catarata, distúrbios da visão de cor, anormalidades pupilares, miopia, alterações do epitélio pigmentar da retina, nistagmo, perda de campo visual e glaucoma $(7,9,12,14,15)$.

\section{DIABETES INSIPIDUS}

O diabetes insipidus na SW é de origem central, podendo ser parcial ou total. Barret e cols. verificaram 
uma prevalência de DI de 73\%, com uma idade média ao diagnóstico de 14 anos (3). Muitas vezes o diagnóstico é retardado pela semelhança da sintomatologia com o diabetes mellitus, sendo a poliúria e a polidipsia atribuídas a um controle inadequado do diabetes mellitus em vez de manifestações do diabetes insipidus. Estudos histopatológicos mostram que o diabetes insipidus ocorre devido a atrofia e gliose do sistema neuro-hipofisário $(14,16,17)$.

\section{SURDEZ}

A perda auditiva é de origem neurossensorial bilateral, envolvendo inicialmente sons de alta freqüência com perda progressiva até as baixas freqüências. A prevalência de perda auditiva ou audiograma anormal tem sido reportada entre 39 a $100 \%(3,15,18)$, sendo, porém, clinicamente evidente em apenas $12 \%$ dos casos. A perda auditiva inicia-se nas três primeiras décadas de vida, é progressiva, mas poucos pacientes tornam-se completamente surdos (19). Muitos pacientes se beneficiam de próteses auditivas.

\section{ANORMALIDADES DO TRATO URINÁRIO}

As anormalidades urológicas presentes na SW são diversas e incluem graus variáveis de dilatação do trato urinário superior e disfunção vesical. A disfunção vesical mais comumente observada é uma bexiga atônica com grande capacidade $(3,7,8,15,20$ 22). Tekgul e col. (21) reportaram que bexiga com baixa capacidade e dissinergia de esfíncter são também manifestações comuns. Alguns autores atribuem a dilatação das vias urinárias ao fluxo aumentado devido ao diabetes insipidus e melhora dessa condição com o uso de hormônio antidiurético (23). Entretanto, há varias descrições de SW com dilatação das vias urinárias sem a presença de diabetes insipidus, indicando ser essa condição resultante primariamente de uma degeneração neural $(10,11,19)$. Chu e cols. (24), através de estudo imuno-histológico de parede vesical e ureter, identificaram uma diminuição importante de fibras nervosas. Uma vez que não há grandes séries de pacientes com avaliação urológica detalhada, é provável que a prevalência de anormalidades urológicas na SW seja subestimada. Tekgul e col. submeteram os pacientes a uma avaliação urológica completa e reportaram que $78,5 \%$ apresentavam vários graus de hidronefrose e $93 \%$ alguma forma de disfunção vesical (21).

\section{COMPLICAÇÕES DO SNC}

Uma variedade de sintomas neurológicos tem sido descrita na SW, e inclui ataxia de tronco gerando marcha instável e quedas, crises de apnéia de origem central, perda de olfato e paladar, hemiparesia devido a infartos cerebrais, mioclonias e nistagmo. Os achados ao exame de ressonância magnética do cérebro são ausência de sinal de alta intensidade da neuro-hipófise, atrofia da região hipotalâmica, cerebelo, tronco e córtex cerebral, além de atrofia do nervo e quiasma óptico (17,25-27). Dados de autópsia mostram uma perda de neurônios com distrofia e edema axonal, freqüentemente associados com gliose e áreas de desmielinização sem sinais de inflamação $(28,29)$.

\section{OUTRAS MANIFESTAÇÕES}

Recentemente, disfunção de hipófise anterior foi reportada na SW. Medlej e col. verificaram secreção anormal de um ou mais hormônios hipofisários em $75 \%$ dos pacientes, sendo a deficiência de GH a alteração mais freqüentemente documentada (30). Interessante é que o hipopituitarismo nesses pacientes não provocou sintomas clínicos, explicando a ausência de investigação de alterações da hipófise anterior em muito estudos prévios.

Hipogonadismo tem sido reportado em pacientes com SW do sexo masculino, tanto devido à disfunção hipotálamo-hipofisária $(15,18)$ quanto de causa primariamente gonadal $(3,15)$. Muitas pacientes do sexo feminino têm menarca atrasada e irregularidades menstruais, porém não há relatos de falência ovariana. Gestações bem sucedidas têm sido reportadas $(3,4,31)$.

Alta prevalência de doenças psiquiátricas tem sido verificada por alguns autores. Em uma revisão de prontuários médicos de 68 pacientes portadores de SW dos Estados Unidos, verificou-se que 60\% apresentavam depressão grave, psicose e outras condições (32). Em alguns casos, as manifestações eram tão importantes que necessitavam de internação em hospital psiquiátrico. Observou-se, ainda, que indivíduos carreadores em heterozigose de mutações no gene da SW são também predispostos a doenças psiquiátricas (33).

\section{PREVALÊNCIA}

A prevalência estimada da SW é de 1 em 100.000 a 770.000 indivíduos, e a prevalência de carreadores de 1 em 100 a 354 indivíduos $(3,34,35)$. 


\section{BASES MOLECULARES}

O gene WFS-1 ou wolframina consiste de 8 exons e estende-se por 33,4 kbases do DNA genômico. O início da tradução ocorre no segundo exon e origina um peptídeo de 890 aminoácidos com um peso molecular de $100 \mathrm{kDa}$, denominado proteína wolframina $(31,43,44)$. Essa proteína apresenta três domínios estruturais: uma região hidrofílica amino-terminal de aproximadamente 300 resíduos, uma região hidrofílica carboxi-terminal de aproximadamente 240 resíduos e uma região central hidrofóbica de aproximadamente 350 resíduos que contém 9-10 segmentos transmembrana (43) (figura 2). O perfil de expressão da proteína foi feito utilizando-se anticorpos contra as porções amino e carboxi terminal e verificou-se que a wolframina é abundantemente expressa em pâncreas, cérebro, coração e músculo; menores quantidades são presentes em fígado e baixos níveis em rins e baço (46). No pâncreas, é primariamente localizada nas células $\beta$, presente em baixos níveis nas células a e ausente no tecido exócrino (47). Estudos bioquímicos mostram que a wolframina é uma glicoproteína de membrana, sensível à endoglicosidase $\mathrm{H}$ e de localização no retículo endoplasmático, apesar de não ser claro se esse representa seu único sítio de localização (48).

A função da wolframina ainda não está completamente definida, porém sua localização sugere um papel na regulação da homeostase do cálcio no retículo endoplasmático, transporte de membrana ou processamento protéico (48). De fato, Osman e col. demonstraram que a superexpressão da wolframina em oócitos de Xenopus

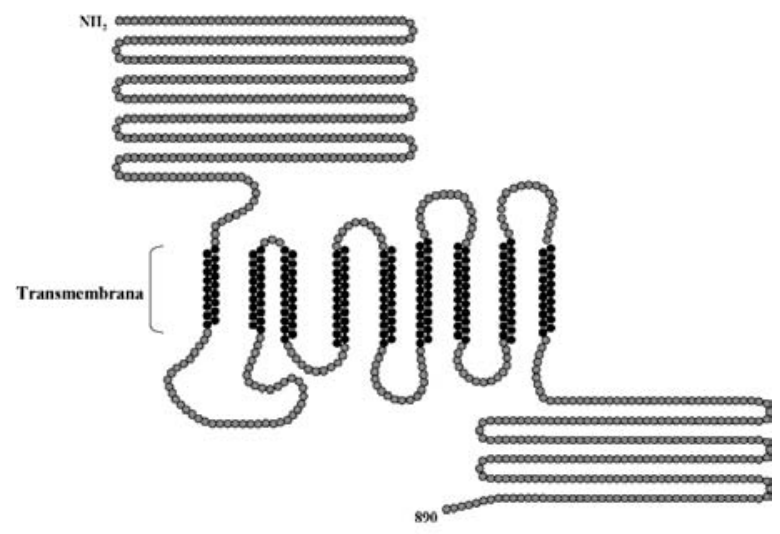

Figura 2. Estrutura hipotética da proteína wolframina demonstrando as porções amino e carboxi terminal e os nove domínios transmembrana. Círculos cinza indicam domínios hidrofilicos e círculos pretos indicam os domínios transmembrana. (Adaptado de Hardy e cols. (52)) levou a um aumento do $\mathrm{Ca}^{++}$citosólico e indução de atividade de canal cátion-seletivo na membrana do retículo endoplasmático (47). Uma vez que a regulação do $\mathrm{Ca}^{++}$pelo retículo endoplasmático é importante na apoptose celular, esses dados sugerem que a wolframina possa estar envolvida na regulação da apoptose mediada pelo retículo endoplasmático, e que um defeito nesse processo possa ser a causa da perda progressiva das células $\beta$ pancreáticas e a degeneração neuronal associadas com a SW. Recentemente, Ishihara e col. demonstraram que camundongos com ruptura do gene WFS- 1 apresentam perda progressiva das células $\beta$ e aumento de apoptose (49). Por outro lado, McBain e col., através do uso de transcripto anti-sense, verificaram que a expressão da wolframina é crítica para o crescimento e proliferação das células $\beta$, e sua expressão reduzida resulta em redução importante na taxa de crescimento, mas não em aumento de apoptose (50).

Entretanto, apesar da função exata da wolframina não estar ainda totalmente definida, é certo que mutações em seu gene são causa da SW. Várias mutações em homozigose ou heterozigose composta incluindo missense, nonsense, inserções e deleções foram identificadas na SW. Essas mutações podem ser identificadas em cerca de $90 \%$ dos indivíduos afetados $(5,51$ 53 ) e são distribuídas ao longo de toda seqüência codificadora, porém muitas dessas mutações são localizadas no exon $8(5,53)$. Não há uma clara relação fenótipogenótipo na SW, porém alguns autores referem um fenótipo mais grave nos pacientes com proteína truncada e mutação na porção carboxi-terminal associada com surdez (53), ou ainda a presença de mutações inativadoras em ambos os alelos seriam associadas com um início mais precoce do diabetes mellitus (5).

Em conclusão, o curso clínico da SW é variável e o entendimento de como alterações na função da wolframina resultam em diabetes e neurodegeneração é essencial para o desenvolvimento de terapias para prevenir ou atenuar as conseqüências dessa doença tão devastadora.

\section{REFERÊNCIAS}

1. Inukai K, Awata $\mathrm{T}$, Inoue $\mathrm{K}$, Kuihara S, Nakashima $\mathrm{Y}$, Watanabe $M$, et al. Identification of a novel WFS1 mutation (AFF344-345ins) in Japanese patients with Wolfram syndrome. Diabetes Res Clin Pract 2005;69:136-41.

2. Wolfram DJ, Wagner HP. Diabetes mellitus and simple optic atrophy among siblings: report of four cases. Mayo Clinic Proc 1938:13:715-8.

3. Barrett TG, Bundey SE, Macleod AF. Neurodegeneration and diabetes: UK nationwide study of Wolfram (DIDMOAD) syndrome. Lancet 1995;346:1458-63. 
4. Kinsley BT, Swift M, Dumont RH, Swift RG. Morbidity and mortality in the Wolfram syndrome. Diabetes Care 1995; 18:1566-70.

5. Giuliano F, Bannawarth S, Mannot S, Cano A, Chabrol B, Vialettes B, et al. Wolfram syndrome in French population: Characterization of novel mutations and polymorphism in the WFS1 Gene. Hum Mutat 2005;25:99-100.

6. Karasik A, O'Hara C, Srikanta S, Swift M, Soeldner JS, Kahn $\mathrm{CR}$, et al. Genetically programmed selective islet $\beta$-cell loss in diabetic subjects with Wolfram's syndrome. Diabetes Care 1989:12:135-8.

7. Blasi C, Pierelli F, Rispoli E, Sapanora M, Vingob E, Andreani D. Wolfram's syndrome: a clinical, diagnostic and interpretative contribution. Diabetes Care 1986 9:521-8.

8. Blasi C, Lulli $P$, Andreani D. HLA antigens in Wolfram (DIDMOAD) syndrome. Lancet 1983;ii:573.

9. Fishman L, Ehrlich RM. Wolfram syndrome: report of four cases and review of literature. Diabetes Care 1986; 9:405-8.

10. Reis AF, Ferreira JG, Coifman R, Russo EMK, Moisés RCS, Dib SA. Síndrome de Wolfram: Relato de um caso, revisão da literatura e caracterização do diabetes mellitus associado. Arq Bras Endocrinol Metab 1996:40:106-12.

11. Megighian D, Savastano M. Wolfram syndrome. Int J Pediatr Otorhinolryngol 2004;68:243-7.

12. Fontes BM, Chen J, Príncipe AH, Sallum JMF, Chicani CF. Síndrome de Wolfram - Relato de caso. Arq Bras Oftalmol 2004;67:961-4.

13. Cryns K, Pfister M, Pennings RJE, Bom SJH, Flothmann K, Caethoven G, et al. Mutations in the WFS1 Gene that causes low-frequency sensorineural hearing loss are small non-inactivating mutations. Hum genet 2002; 110:389-94.

14. Al-Till M, Jarrah NS, Ajlouni KM. Ophthalmologic findings in fifteen patients with Wolfram syndrome. Eur J Ophtalmol 2002;12:84-8.

15. Cremers CWR, Wijdeveld PGAB, Pinckers AJLG. Juvenile diabetes mellitus, optic atrophy, hearing loss, diabetes insipidus, atonia of the urinary tract and bladder and other abnormalities (Wolfram syndrome). Acta Paediatr Scand 1997;264(suppl.):1-16.

16. Mayer UM, Rott HD, Böhles HJ. Observation concerning the age of onset and the nature of optic atrophy in Wolfram's syndrome (DIDMOAD). Ophthalmic Paediatr Genet 1985;3:155-7.

17. Barret TG, Bundey SE, Fielder AR, Good PA. Optic atrophy in Wolfram (DIDMOAD) syndrome. Eye $1997 ; 11: 882-8$

18. Simsek E, Simsek T, Tekgül S, Hosal S, Seyrantepe V, Aktan G. Wolfram (DIDMOAD) syndrome: a multidisciplinary clinical study in nine Turkish patients and review of the literature. Acta Paediatr 2003:92:55-61.

19. Dreyer M, Rüdiger HW, Bujara K, Herberhold C, Kühnau J, Maack P, et al. The syndrome of Diabetes Insipidus, Diabetes Mellitus, Optic Atrophy, Deafness, and other abnormalities (DIDMOAD-Syndrome): Two affected sibs and a short review of literature (98 cases). Klin Wochenschr 1982:60:471-5.
20. Kinsely BT, Firth RG. The Wolfram syndrome: a primary neurodegenerative disorder with lethal potential. Ir Med J 1992;85:34-6

21. Tekgul S, Oge O, Simsek E, Yordam N, Kendi S. Urological manifestations of the Wolfram syndrome: observations in 14 patients. J Urol 1999;161:616-7.

22. Najjar SS, Saiklay MG, Zayłoun GM, Abdelmooz A. Association of diabetes insipidus, diabetes mellitus, optic atrophy and deafness: the Wolfram or DIDMOAD syndrome. Arch Dis Child 1985;60:823-8.

23. Page $M M c B$, Asmal AC, Edwards CR. Recessive inheritance of diabetes: the syndrome of diabetes insipidus, diabetes mellitus, optic atrophy and deafness. Q J Med 1976; 179:505-20.

24. Chu P, Staff WG, Morris JA, Polak JM. DIDMOAD syndrome with megacystic and megaureter. Postgrad Med J 1986:62:859-63.

25. Scolding NJ, Keller-Wood H, Shaw C, Shneerson J, Antoun N. Wolfram syndrome: hereditary diabetes mellitus with brainstem and optic atrophy. Ann Neurol 1996;39:352-60.

26. Rando TA, Horton JC, Layzer RB. Wolfram syndrome: evidence of a diffuse neurodegenerative disease by magnetic resonance imaging. Neurology 1992; 42:1220-4.

27. Alicangolu R, Canbakan B, Yldiz N, Arikan E, Kundur $\mathrm{H}$ Bahtiyar $\mathrm{K}$, et al. DIDMOAD syndrome. Wien Med Wochenschr 1994; 144:78-81.

28. Shannon P, Becker L, Deck J. Evidence of widespread axonal pathology in Wolfram syndrome. Acta Neuropathol 1999:98:304-8.

29. Gregorios JB. Wolfram's syndrome with schizophrenia and central hypoventilation: a neuropathological study. J Neuropathol Exp Neurol 1989;48:308-10.

30. Medlej R, Wasson P, Baz P, Azar S, Salti S, Loiselet J, et al. Diabetes mellitus and optic atrophy: A study of Wolfram syndrome in the Lebanese population. J Clin Endocrinol Metab 2004;89:1656-61.

31. Minton JAL, Rainbow LA, Ricketts C, Barrett TG. Wolfram syndrome. Rev Endocr Metab Disord 2003;4:53-9.

32. Swift RG, Sadler DB, Swift M. Psychiatric findings in Wolfram syndrome homozygotes. Lancet 1990;336:667-9.

33. Swift RG, Perkins DO, Chase CL, Sadler DB, Swift M. Psychiatric disorders in 36 families with Wolfram syndrome. J Psychiatry 1991;148:775-9.

34. Fraser F, Gunn T. Diabetes mellitus, diabetes insipidus and optic atrophy: An autosomal recessive syndrome? J Med Genet 1977; 14:190-3.

35. Gunn T, Bortolussi R, Little JM, Andermann F, Fraser FC, Belmonte MM. Juvenile diabetes mellitus, optic atrophy, sensory nerve deafness, and diabetes insipidus - a syndrome. J Pediatr 1976;89:565-70.

36. Bundey S, Poulton K, Whitewell H, Curtis E, Brown IA, Fielder AR. Mitochondrial abnormalities in the DIDMOAD syndrome. J Inherit Metab Dis 1992;15:315-9.

37. Bundey S, Fielder AR, Poulton K. Wolfram syndrome: Mitochondrial disorder. Lancet 1993:342:1059-60. 
38. Bu X, Rotter JL. Wolfram syndrome: A mitochondrial-mediated disorder? Lancet 1993:342:598-600.

39. Vora AJ, Lilleyman JS. Wolfram syndrome: Mitochondrial disorder. Lancet 1993;342:1059.

40. Barrientos A, Casademont J, Cardellach F, Volpini V, Solans A, Tolosa $\mathrm{E}$, et al. Autossomal recessive Wolfram syndrome associated with an 8.5-kb mtDNA single deletion. Am J Hum Genet 1996;58:963-70.

41. Rotig A, Cormer V, Chatelain P, Francois R, Saudubray $\mathrm{JM}$, Rustin $\mathrm{P}$, et al. Deletion of mitochondrial DNA in a case of early-onset diabetes mellitus, optic atrophy, and deafness (Wolfram syndrome, MIM 222300). J Clin Invest 1993:91:1095-8.

42. Polymeropoulos MH, Swift RG, Swift M. Linkage of thr gene for Wolfram syndrome to markers on short arm of cromossome 4. Nat Genet 1994;8:95-7.

43. Inoue H, Tanizawa Y, Wasson J, Behn P, Kalidas K, BernalMizrachi $\mathrm{E}$, et al. A gene encoding a transmembrane protein is mutated in patients with diabetes mellitus and optic atrophy (Wolfram syndrome). Nat Genetics 1998;20:143-8

44. Strom TM, Hortnagel K, Hofmann S, Gekeler F, Scharfe C, Rabl W, et al. Diabetes insipidus, diabetes mellitus, optic atrophy and deafness (DIDMOAD) caused by mutations in a novel gene (wolframin) coding for a predicted transmembrane protein. Hum Molec Genet 1998;7:2021-8.

45. El-Shanti H, Lidral AC, Jarrah N, Druhan L, Ajlouni K. Homozygosy mapping identities and additional locus for Wolfram syndrome on chromosome 4q. Am J Hum Genet 2000;66:1229-36.

46. Hofman S, Philbrook C, Gerbitz KD, Bauer MF. Wolfram syndrome: structural and functional analyses of mutant and wild-type wolframin, the WFS1 product. Hum Mol Genet 2003; 12:2003-12.

47. Osman AA Saito M Makepeace C, Permutt MA Schlesinger $\mathrm{P}$, Mueckler $\mathrm{M}$. Wolframin expression induces novel ion channel activity in endoplasmic reticulum membranes and increases intracellular calcium. J Biol Chem 2003:278:52755-62.
48. Takeda K, Inoue H, Tanizawa Y, Matsuzaki Y, Oba J, Watanabe $Y$, et al. WFS1 (Wolfram syndrome 1) gene product: predominant subcellular localization to endoplasmatic reticulum in cultured cells and neuronal expression in rat brain. Hum Mol Genet 2001:10:477-84

49. Ishihara H, Tamura A, Takahashi $\mathrm{R}$, Inoue H, Tanizawa $\mathrm{Y}$, Takeda S, et al. Disruption of the WFS1 gene causes diabetes in mice due to progressive loss of islet $\beta$-cells. Diabetes 2003:52(suppl. 1):A42.

50. McBain S, Morgan NG. Functional effects of expression of wolframin-antisense transcripts in BRIN-BD1 $1 \beta$-cells. Biochem Biophys Res Commun 2003;307:684-8.

51. Colosimo A, Guida V, Rigoli L, Di-Bella C, De-Luca A, Briuglia $S$, et al. Molecular detection of novel WFS1 mutations in patients with Wolfram syndrome by a DHPLC-based assay. Hum Mutat 2003:21:622-9.

52. Hardy C, Khanin F, Torres R, Scott-Brown M, Seller A, Poulton $\mathrm{J}$, et al. Clinical and molecular genetic analyses of 19 Wolfram syndrome kindreds demonstrating a wide spectrum of mutations in WFS1. Am J Hum Genet 1999:65:1279-90.

53. Smith CJA, Crock PA, King BR, Meldrum CJ, Scott RJ, Phenotype-genotype correlations in a series of Wolfram syndrome families. Diabetes Care 2004:27:2003-9.

\section{Endereço para correspondência:}

Regina S. Moisés

Disciplina de Endocrinologia

Universidade Federal de São Paulo

Rua Botucatu 740, $2^{\circ}$ andar

04034-970 São Paulo, SP

Fax: (11) 5579-6636

E-mail: rmoises@endocrino.epm.br 